\title{
INTRODUÇÃO E SELEÇÃO DE ESPÉCIES ARBÓREAS FORRAGEIRAS EXÓTICAS NA REGIÃO SEMI-ÁRIDA DO ESTADO DE SERGIPE'
}

\author{
Marcos Antônio Drumond ${ }^{2}$ \\ Orlando Monteiro de Carvalho Filho ${ }^{2}$ \\ Visêldo Ribeiro de Oliveira ${ }^{2}$
}

\section{Recebido em 21/12/1998. Aceito em 29/07/1999}

\begin{abstract}
RESUMO - (Introdução e seleção de espécies arbóreas forrageiras exóticas na região semi-árida do Estado de Sergipe). Este trabalho teve como objetivo selecionar espécies arbóreas de uso madeireiro e forrageiro para a região semi-árida do Estado de Sergipe. Foram introduzidas quinze espécies procedentes da região semi-árida da América Central: Acacia famesiana, Albizia caribaea, Albizia guachepele, Ateleia herbert-smithii, Caesalpinia coriaria, Caesalpinia eryostachys, Caesalpinia velutina, Enterolobium cyclocarpum, Gliricidia sepium, Leucaena diversifolia, Leucaena leucocephala, Leucaena shannoni, Parkinsonia aculeata, Pithecellobium dulce e Senna otomaria. As espécies estudadas foram plantadas no Campo Experimental de Glória, da Embrapa Semi-Árido, no município de Nossa Senhora da Glória, SE. O plantio foi feito em covas de 30x30cm, sem adubação, espaçadas em $3 \times 2 \mathrm{~m}$, seguindo o delineamento estatístico de blocos ao acaso com três repetições, com 36 plantas por parcela. Foram avaliadas a sobrevivência, a altura e o diâmetro à altura de $1,30 \mathrm{~m}$ do solo aos 55 meses de idade após o plantio definitivo no campo. Os resultados mostram que as espécies $G$. sepium e $A$. guachepele sobressaíram-se em relação às demais, apresentando, respectivamente, alturas médias ( \pm desvio-padrão) de $4,0 \pm 0,4 \mathrm{e} 4,3 \pm 0,2 \mathrm{~m}$, diâmetros médios ( \pm desviopadrão) de 4,4 $\pm 0,5$ e $6,3 \pm 0,1 \mathrm{~cm}$, com taxas de sobrevivência de 100 e $98 \%$. L leucocephala, embora tenha atingido altos valores médios de altura $(5,0 \pm 0,3 \mathrm{~m})$ e de diâmetro $(6,0 \pm 1,5 \mathrm{~cm})$, apresentou baixa taxa de sobrevivência (44\%). Ressalta-se, ainda, a mortalidade de $100 \%$ dos indivíduos de $L$ diversifolia e A. farnesiana. As espécies Gliricidia sepium e Albizia guachepele destacaram-se silviculturalmente, com grande potencialidade para áreas semi-áridas do Estado de Sergipe. Acacia farnesiana, Leucaena diversifolia e Senna otomaria não se adequaram às condições semi-áridas da região.
\end{abstract}

Palavras-chave - Gliricidia sepium, Albizia guachepele, Leucaena leucocephala, árvores de uso múltiplo, semi-árido, silvicultura

\begin{abstract}
Introduction and selection of arboreal forage species in the semi-arid region of Sergipe State). The objective of this work was the selection of arboreal species for multiple use in the semi-arid area of the state of Sergipe, Brazil. Fifteen species from the semi-arid area of Central America were introduced in the region: Acacia farnesiana, Albizia caribaea, Albizia guachepele, Ateleia herbert-smithii, Caesalpinia coriaria, Caesalpinia eryostachys, Caesalpinia velutina, Enterolobium cyclocarpum, Gliricidia sepium, Leucaena diversifolia, Leucaena leucocephala, Leucaena shannoni, Parkinsonia aculeata, Pithecellobium dulce and Senna otomaria. The species were planted in the Experimental Field of Glória, Embrapa Semi-Árido, in Nossa Senhora da Glória, Sergipe State, in holes of $30 \mathrm{~cm} \times 30 \mathrm{~cm}$, without fertilization, $3.0 \mathrm{~m} \times 2.0 \mathrm{~m}$ apart in a randomized block design with three replications and 36 plants per plot. They were evaluated for survival, height, diameter at the height of $1.30 \mathrm{~m}$ from the soil surface, 55 months after planting. G. sepium and A. guachepelle showed higher growth than the other species, with mean heights ( \pm standard deviation), of $4.0 \pm 0.4$ and $4.3 \pm 0.2 \mathrm{~m}$ respectively, and mean diameters ( \pm standard deviation) of $4.4 \pm 0.5$ and $6.3 \pm 0.1 \mathrm{~cm}$, and survival rates of 100 and $98 \%$. Although $L$. leucocephala showed excellent growth in height $(5.0 \pm 0.3 \mathrm{~m})$ and in diameter $(6.0 \pm 1.5 \mathrm{~cm})$, its survival rate was $44 \% . L$. diversifolia and A. farnesiana have shown 100\% mortality in the studied plots. Gliricidia sepium and Albizia guachepele were outstanding, with great potentiality for the semi-arid areas in the State of Sergipe. Acacia farnesiana, Leucaena diversifolia and Senna otomaria did not show a good performance in the semi-arid conditions of the region.
\end{abstract}

Key words - Gliricidia sepium, Albizia guachepele, Leucaena leucocephala, multipurpose trees, semi-arid, silviculture

1 Trabalho apresentado no $49^{\circ}$ Congresso Nacional de Botânica, Salvador, BA

2 EMBRAPA, Semi-Árido, BR 428, km 152, C. Postal 23, CEP 56300-970, Petrolina, PE, Brasil e-mail: drumond@cpatsa.com.br 


\section{Introdução}

O semi-árido brasileiro, ocupando área de aproximadamente $900.000 \mathrm{~km}^{2}$, é caracterizado pelo elevado déficit hídrico e má distribuição das chuvas. A temperatura média é de $26^{\circ} \mathrm{C}$ com umidade relativa entre 50 e $70 \%$ e insolação média de 2.800 horas/ano (Embrapa 1979). Os solos são geralmente rasos, chegando a apresentar afloramentos rochosos, reduzida capacidade de retenção de água e baixos teores de nutrientes e matéria orgânica.

Com pequena diversidade de espécies arbóreas e arbustivas, a vegetação denominada caatinga é caracterizada pela baixa capacidade de produção de lenha e forragem. Segundo Albuquerque (1988) e Guimarães Filho \& Soares (1988), a capacidade de suporte forrageiro da caatinga é de 13 a 14 ha/bovino adulto e de 1 a 1,3 ha/caprino. A produção madeireira varia de 7,3 a $58,0 \mathrm{~m}^{3} / \mathrm{ha}$ (Tavares et al. 1969; 1970; Carvalho 1971; Lima et al. 1978). Muitas espécies, além da importância madeireira e forrageira, são ainda utilizadas na alimentação humana e na medicina caseira.

Apesar da ampla utilização dessas espécies, existe grande demanda por recursos florestais em propriedades onde cada vez mais as árvores assumem importante papel na economia rural. No entanto, o número de espécies de valor econômico indicado para reflorestamento na região é limitado e as espécies possuem baixa produtividade. Desta forma, é importante a utilização de espécies de uso múltiplo, isto é, aquelas cultivadas, mantidas ou manejadas preferencialmente para mais de uma finalidade específica, que venham a aumentar a disponibilidade, principalmente, de madeira e forragem na região. A utilização dessas espécies contribuirá indiretamente à preservação das espécies nativas da caatinga.

Este trabalho teve como objetivo selecionar espécies arbóreas de uso múltiplo que possam ter cultivo viável na região semi-árida do Estado de Sergipe.

\section{Material e métodos}

O trabalho foi desenvolvido no Campo Experimental de Glória, da Embrapa Semi-Árido, em Nossa Senhora da Glória, Sergipe (10^13’ S, $37^{\circ} 27^{\prime}$ 'W; altitude $\left.300 \mathrm{~m}\right)$. A precipitação média anual varia em torno de $659 \mathrm{~mm}$, com predominância de chuvas nos meses de abril a agosto. A temperatura média anual é de $24,7^{\circ} \mathrm{C}$ variando entre a mínima $17^{\circ} \mathrm{Ce}$ a máxima de $37^{\circ} \mathrm{C}$. Os solos são do tipo Podzólico vermelho amarelo eutrófico, com pH em torno de 5,8; P=1,0ppm e K=129ppm.

Oexperimento, em delineamento estatístico de blocos ao acaso com 15 tratamentos (Acacia farnesiana (L.) Willd., Albizia caribaea (Urb.) Britt \& Rose, Albizia guachepele (Kuinth.) Gug., Ateleia herbert-smithii Pittier, Caesalpinia coriaria (Jacq.) Willd., Caesalpinia eryostachys Benth., Caesalpinia velutina (Britt \& Rose) Standl., Enterolobium cyclocarpum (Jacq.) Criseb, Gliricidia sepium (Jacq.) Steud, Leucaena diversifolia (Schlecht.) Benth., Leucaena leucocephala Lam De Wit, Leucaena shannoni Donn-Smith, Parkinsonia aculeata L., Pithecellobium dulce (Roxb.) Benth. e Senna otomaria (L.) Irvin and Barneby), três repetições, foi constituído de parcelas retangulares de $216 \mathrm{~m}^{2}(12 \times 18 \mathrm{~m})$, ocupando área total de $9.720 \mathrm{~m}^{2}$. Cada parcela foi constituída de 36 plantas por espécie, em covas de $30 \times 30 \mathrm{~cm}$, sem adubação, em espaçamento de $3 \times 2 \mathrm{~m}$.

As plantas foram propagadas por sementes obtidas através do Oxford Forestry Institute - OFI, procedentes da América Central (Tab. 1), sendo avaliada a sobrevivência aos $7,12,26,38$ e 55 meses. A altura e o DAP (diâmetro a $1,30 \mathrm{~m}$ do solo) foram avaliados aos 55 meses de idade, não considerando plantas com DAP inferior a $1,5 \mathrm{~cm}$. Os dados foram submetidos à análise de variância e as médias, comparadas pelo teste de Tukey, ao nível de 5\% de probabilidade. Como forma de manejo, foram efetuados anualmente apenas os tratos silviculturais de manutenção, sendo uma capina ao início do período chuvoso e outra ao final.

\section{Resultados e discussão}

Aos sete meses de idade, à exceção de Senna otomaria (com $100 \%$ de mortalidade), a sobrevivência das espécies foi acima dos $80 \%$. Entretanto, após 55 meses, em função do manejo e condições climáticas locais, as espécies melhor aclimatadas se sobressaíram significativamente em relação às demais, quanto à sobrevivência: Gliricidia sepium 
Tabela 1. Origem das espécies arbóreas de uso múltiplo testadas na região semi-árida do Estado de Sergipe.

\begin{tabular}{|c|c|c|c|c|c|c|}
\hline Espécies & Local & País & $\begin{array}{c}\text { Lat. } \\
\mathrm{N}\end{array}$ & $\begin{array}{c}\text { Long. } \\
\text { W }\end{array}$ & $\begin{array}{l}\text { Alt. } \\
(\mathrm{m})\end{array}$ & $\begin{array}{l}\text { Prec. } \\
(\mathrm{mm})\end{array}$ \\
\hline Acacia farnesiana & Llanos de la Fragua & Guatemala & $14^{\circ} 59^{\prime}$ & $89^{\circ} 40^{\prime}$ & 200 & 625 \\
\hline Albizia caribaea & La Cruz & Honduras & $13^{\circ} 23^{\prime}$ & $87^{\circ} 13^{\prime}$ & 100 & 1120 \\
\hline Albizia guachepele & Motagua Valley & Guatemala & $14^{\circ} 59^{\prime}$ & $89^{\circ} 30^{\prime}$ & 200 & 625 \\
\hline Ateleia herbert-smithii & El Bordo las Tinajas & Nicarágua & $12^{\circ} 43^{\prime}$ & $86^{\circ} 18^{\prime}$ & 400 & 922 \\
\hline Caesalpinia coriaria & Las Maderas & Nicarágua & $12^{\circ} 25^{\prime}$ & $86^{\circ} 03^{\prime}$ & 60 & 1184 \\
\hline Caesalpinia eryostachys & Tapaire & Honduras & $13^{\circ} 32^{\prime}$ & $87^{\circ} 07^{\prime}$ & 130 & 1119 \\
\hline Caesalpinia velutina & El Rancho & Guatemala & $14^{\circ} 57^{\prime}$ & $90^{\circ} 01^{\prime}$ & 250 & 526 \\
\hline Enterolobium cyclocarpum & Jesus de Otoro & Honduras & $14^{\circ} 28^{\prime}$ & $88^{\circ} 00^{\prime}$ & 650 & 915 \\
\hline Gliricidia sepium & Laguna & Nicarágua & $12^{\circ} 37^{\prime}$ & $86^{\circ} 03^{\prime}$ & 380 & 922 \\
\hline Leucaena diversifolia & Puerto de Golpe & Guatemala & $15^{\circ} 02^{\prime}$ & $89^{\circ} 40^{\prime}$ & 480 & 724 \\
\hline Leucaena leucocephala & San Felipe & Honduras & $13^{\circ} 38^{\prime}$ & $86^{\circ} 55^{\prime}$ & 1050 & 750 \\
\hline Leucaena shannoni & Comayagua Valley & Honduras & $14^{\circ} 22^{\prime}$ & $87^{\circ} 39^{\prime}$ & 650 & 960 \\
\hline Parkinsonia aculeata & Rio San Antonio & Nicarágua & $12^{\circ} 23^{\prime}$ & $86^{\circ} 09^{\prime}$ & 55 & 1184 \\
\hline Pithecellobium dulce & San Ramon & Nicarágua & $12^{\circ} 19^{\prime}$ & $86^{\circ} 14^{\prime}$ & 50 & 1184 \\
\hline Senna otomaria & Valle Comayagua & Honduras & $14^{\circ} 22^{\prime}$ & $87^{\circ} 39^{\prime}$ & 650 & 1020 \\
\hline
\end{tabular}

Tabela 2. Taxa de sobrevivência das espécies arbóreas de uso múltiplo, em diferentes idades, em Nossa Senhora da Glória, Sergipe.

\begin{tabular}{|c|c|c|c|c|c|}
\hline \multirow{2}{*}{ Espécies } & \multicolumn{5}{|c|}{ Sobrevivência (\%) } \\
\hline & 7 meses & 12 meses & 26 meses & 38 meses & 55 meses \\
\hline Acacia farnesiana & 97 & 91 & 82 & 0 & 0 \\
\hline Albizia caribaea & 84 & 83 & 83 & 83 & $59 \mathrm{c}$ \\
\hline Albizia guachepele & 98 & 98 & 98 & 98 & $98 \mathrm{a}$ \\
\hline Ateleia herbert-smithii & 100 & 100 & 100 & 98 & 94 a \\
\hline Caesalpinia coriaria & 96 & 96 & 96 & 96 & $96 \mathrm{a}$ \\
\hline Caesalpinia eryostachys & 91 & 91 & 88 & 63 & $63 c$ \\
\hline Caesalpinia velutina & 86 & 82 & 82 & 79 & $62 \mathrm{c}$ \\
\hline Enterolobium cyclocarpum & 94 & 90 & 88 & 84 & $81 \mathrm{~b}$ \\
\hline Gliricidia sepium & 100 & 100 & 100 & 100 & $100 \mathrm{a}$ \\
\hline Leucaena diversifolia & 92 & 50 & 13 & 6 & 0 \\
\hline Leucaena leucocephala & 100 & 94 & 73 & 71 & $44 \mathrm{~d}$ \\
\hline Leucaena shannoni & 94 & 81 & 81 & 81 & $56 \mathrm{~cd}$ \\
\hline Parkinsonia aculeata & 97 & 94 & 94 & 94 & $94 \mathrm{a}$ \\
\hline Pithecellobium dulce & 98 & 98 & 98 & 98 & $98 \mathrm{a}$ \\
\hline Senna otomaria & 0 & 0 & 0 & 0 & 0 \\
\hline
\end{tabular}

Valores seguidas de mesma letra na coluna não diferem entre si pelo teste de Tukey ao nível de $5 \%$ de probabilidade

(100\%), Albizia guachepele (98\%), Pithecellobium dulce (98\%), Caesalpinia coriaria (96\%), Ateleia herbert-smithii $(94 \%)$ e Parkinsonia aculeata (94\%). As demais tiveram sobrevivência significativamente inferior (ao nível de $5 \%$ de probabilidade pelo teste de Tukey; Tab. 2).

Acacia farnesiana, Leucaena diversifolia e Senna otomaria foram eliminadas das análises do ensaio por apresentarem $100 \%$ de mortalidade aos 55 meses após plantio. Albizia caribaea, Caesalpinia coriaria, C. eryostachys e Pithecellobium dulce não foram incluídas na análise de DAP por não apresentarem, em sua maioria, plantas com diâmetro mínimo pré-estabelecido. Os maiores DAP médios ( \pm desvio-padrão) foram observados em Albizia guachepele $(6,3 \pm 0,1 \mathrm{~cm})$ e Leucaena leucocephala $(6,0 \pm 1,5 \mathrm{~cm})$, não diferindo estatisticamente de Gliricidia sepium $(4,4 \pm 0,4 \mathrm{~cm}), L$. shannoni $(4,0 \pm 1,0 \mathrm{~cm})$, Ateleia herber-smithii $(3,9 \pm 0,3 \mathrm{~cm})$ e Enterolobium cyclocarpum $(3,9 \pm 1,0 \mathrm{~cm})$ (Tab. 3).

Quanto à altura, o maior valor médio ( \pm desvio-padrão) foi encontrado para Leucaena leucocephala $(5,0 \pm 0,3 \mathrm{~m})$, não diferindo estatisti- 
Tabela 3. Valores médios ( \pm desvio padrão) de altura, diâmetro e volume de diversas espécies arbóreas de uso múltiplo, em Nossa Senhora da Glória, Sergipe, aos 55 meses de idade.

\begin{tabular}{lcccc}
\hline Espécie & Altura $(\mathrm{m})$ & Diâmetro $(\mathrm{cm})$ & Volume $\left(\mathrm{m}^{3} / \mathrm{ha}\right)$ \\
\hline Albizia guachepele & $4,3 \pm 0,2(47)$ ab & $6,3 \pm 0,2(47)$ a & $21,9 \pm 0,7(47)$ \\
Leucaena leucocephala & $5,0 \pm 0,3(21)$ a & $6,0 \pm 1,5(21)$ a & $10,4 \pm 2,2(21)$ \\
Gliricidia sepium & $4,0 \pm 0,4(48)$ ab & $4,4 \pm 0,5(48)$ ab & $10,1 \pm 2,6(48)$ \\
Ateleia herbert-smithii & $2,9 \pm 0,2(45)$ bc & $3,9 \pm 0,3(45)$ ab & $5,4 \pm 1,3(45)$ \\
Leucaena shannoni & $3,6 \pm 1,0(27)$ ab & $4,0 \pm 1,0(27)$ ab & $4,2 \pm 2,3(27)$ \\
Enterolobium cyclocarpum & $1,9 \pm 1,2(39)$ bc & $3,9 \pm 1,0(39)$ ab & $3,1 \pm 2,9(39)$ \\
Parkinsonia aculeata & $2,4 \pm 0,6(45)$ bc & $2,5 \pm 0,4(45)$ b & $1,8 \pm 0,7(45)$ \\
Caesalpinia velutina & $2,2 \pm 0,6(30)$ bc & $2,9 \pm 0,4(30)$ b & $1,9 \pm 1,2(30)$ \\
Albizia caribaea & $1,0 \pm 0,1(28)$ c & - & - \\
Caesalpinia coriaria & $1,5 \pm 0,3(46)$ c & - & - \\
Caesalpinia eryostachys & $1,0 \pm 0,4(30)$ c & - & - \\
Pitthecellobium dulce & $2,2 \pm 0,2(47)$ c & - & - \\
\hline
\end{tabular}

Médias seguidas de mesma letra nas colunas não diferem entre si pelo teste de Tukey, ao nível de $5 \%$ de probabilidade; valores entre parênteses referem-se ao número de observaçōes que deram origem à média

camente de Albizia guachepele $(4,3 \pm 0,2 \mathrm{~m})$, Gliricidia sepium $(4,0 \pm 0,4 \mathrm{~m})$ e Leucaena shannoni $(3,6 \pm 1,0 \mathrm{~m})$. Menores alturas foram observadas em Caesalpinia coriaria $(1,5 \pm 0,3 \mathrm{~m})$, Caesalpnia eryostachys $(1,0 \pm 0,0 \mathrm{~m})$ e Albizia caribaea $(1,0 \pm 0,1 \mathrm{~m})($ Tab.3).

$\mathrm{Na}$ análise conjunta de sobrevivência, DAP e altura das espécies, sobressaíram Albizia guachepele, Gliricidia sepium e Leucaena leucocephala. Entretanto, Drumond et al. (1998) ao analisar estas mesmas espécies nas condições edafoclimáticas de Petrolina, PE, aos 96 meses de idade, relataram como promissoras Leucaena diversifolia, Ateleia herbert-smithii, Caesalpinia velutina e $C$. coriaria. Nesta idade, foi observada mortalidade total para Gliricidia sepium, Leucaena shannoni, Albizia caribaea e Pithecellobium dulce, que contrastam com os dados de sobrevivência obtidos em Nossa Senhora da Glória.

Segundo Hughes (1988), Gliricidia sepium se desenvolve bem em grande amplitude de condições de solos, com pH variando de 5,5 a 7,0, podendo sobreviver com precipitação de até $400 \mathrm{~mm}$, resistindo a períodos de seca de cinco a seis meses. Picado (1985), utilizando semeio direto, obteve aos quatro anos de idade altura média de 7,0m e 5,9cm de DAP com G. sepium em Cañas, Costa Rica. Nas zonas secas de La Máquina, Guatemala, Martinez (1985) relata valores de $94 \%$ de sobrevivência e $3,1 \mathrm{~m}$ alt. para esta mesma espécie, aos 24 meses de idade. $\mathrm{Na}$
Nicarágua, em Vertissolo, Chan \& Bauer (1986) encontraram $79 \%$ de sobrevivência, $3,3 \mathrm{~cm}$ de DAP e 3,2m alt. em G. sepium, aos 26 meses. Para as condições do nordeste do Brasil, esta espécie necessita ainda de estudos de comportamento nos diferentes ecossistemas da região, face ao seu potencial forrageiro.

Quanto ao gênero Leucaena, a taxa de sobrevivência em L. leucocephala $(44 \%)$, espécie já difundida no semi-árido brasileiro, está abaixo das taxas relatadas para os municípios de Sobral, CE (100\%), Petrolina, PE (100\%), Quixadá, CE (90\%) e em Sumé, PB (76\%) (Silva et al. 1980; Lima 1982). Entretanto em trabalhos de manejo, Lima (1986) concluiu que plantas estabelecidas em espaçamentos mais amplos apresentam maiores índices de sobrevivência. Quanto à $L$. diversifolia e $L$. shannoni, estas são espécies recém introduzidas na região nordeste do Brasil.

As taxas de sobrevivência em Leucaena leucocephala (44\%) e L shannoni (56\%), aos 55 meses de idade, não diferiram estatisticamente entre si pelo teste de Tukey ao nível de $5 \%$ de probabilidade. Já $L$ diversifolia teve mortalidade de 50\% a partir dos 12 meses, chegando a $100 \%$ aos 55 meses. Em Petrolina, PE, Drumond et al. (1988) relatam taxas de sobrevivência de 78 e $31 \%$, respectivamente para $L$ diversifolia e $L$ leucocephala, e $100 \%$ de mortalidade para $L$ shannoni, aos 96 meses de idade. Para as zonas secas da Guatemala, em La Máquina, Martinez (1985) relata 89 e $84 \%$ de 
sobrevivência, 5,4 e 4,6 cm de DAP e 7,9 e 5,6m alt., respectivamente, para Leucaena leucocephala e $L$. diversifolia, aos 27 meses de idade. Em solos do tipo Vertissolo, na Nicarágua, Chan \& Bauer (1986) encontraram média de $90 \%$ de sobrevivência, $3,4 \mathrm{~cm}$ de DAP e 3,3m alt. para L. leucocephala, aos 26 meses de idade.

Quanto ao gênero Caesalpinia, as taxas de sobrevivência de $C$. coriaria $(96 \%)$ foram significativamente maiores pelo teste de Tukey ao nível de $5 \%$ de probabilidade que as de $C$. eryostachys (63\%) e C. velutina (62\%). No estudo de comportamento de espécies em diferentes sítios das zonas secas da Guatemala, Martinez (1986) encontrou $98 \%$ de sobrevivência, $2,9 \mathrm{~cm}$ de DAP e 3,5m alt. em C. velutina na localidade de La Máquina, aos 24 meses de idade; $87 \%$ de sobrevivência, $2,4 \mathrm{~cm}$ de DAP e $3,0 \mathrm{~m}$ alt. em Huité, aos 40 meses de idade; e $6,1 \mathrm{~cm}$ de DAP e $6,4 \mathrm{~m}$ alt. em Gualan, aos 36 meses de idade.

Parkinsonia aculeata e Pithecellobium dulce, que apresentaram sobrevivência superior a $90 \%$, são encontradas no nordeste do Brasil, em arborização urbana. Silva et al. (1980) relatam para $P$. aculeata sobrevivência de $60 \%$ e altura de 4,6m aos 11 anos de idade em Sobral, Ceará. Chan \& Bauer (1986) encontraram $80 \%$ de sobrevivência, $2,7 \mathrm{~cm}$ de DAP e $2,9 \mathrm{~m}$ alt. para $P$. aculeata, em Vertissolo na Nicarágua, aos 26 meses de idade. Goor \& Braney (1976) relatam ainda que $P$. aculeata em solos e climas favoráveis chega a $10 \mathrm{~m}$ alt. em 5 ou 6 anos.

Quanto à Albizia guachepele e Ateleia herbert-smithii, mesmo em suas regiões de origem, são espécies pouco estudadas. Segundo Stewart et al. (1992), Ateleia herbert-smithii é muito utilizada localmente como madeira, lenha e forragem e completamente desconhecida fora de sua zona de ocorrência (área central da Nicarágua e Costa Rica). Quanto à Albizia guachepele, segundo Hughes et al. (1985), aparentemente é espécie de rápido crescimento, resistente à seca. A madeira é utilizada para construção civil e lenha. Na Costa Rica, vem sendo plantada em escala moderada para a produção de madeira (Doland \& Koodriguez 1990 apud Stewart et al. 1992). Face aos dados apresentados no presente trabalho e aos de Drumond et al. (1998) onde A. guachepele apresenta $100 \%$ de mortalidade aos 96 meses de idade em Petrolina, PE, estas espécies requerem maiores estudos para as condições de semi-árido brasileiro.

As espécies Acacia farnesiana, Leucaena diversifolia e Senna otomaria não se adequaram às condições semi-áridas da região. Albizia guachepele, Gliricidia sepium e Leucaena leucocephala foram as espécies que se destacaram, com possibilidades de usos no semi-árido de Sergipe, necessitando, entretanto, de estudos mais aprofundados de comportamento em outros sítios da região.

\section{Agradecimentos}

Ao Oxford Forestry Institute (OFI), pela doação das sementes, que possibilitaram a realização deste trabalho.

\section{Referências bibliográficas}

Albuquerque, S. G. de. 1988. As pastagens do semi-árido do Nordeste. Informe Agropecuário 13(153/154): 40-48.

Carvalho, G. H. de. 1971. Contribuição para a determinação da reserva madeireira do Sertão Central do Estado do Pernambuco. Boletim de Recursos Naturais 9: 289-312, 1971 .

Chan, B. \& Bauer, J. 1985. Comportamiento inicial de seis especies forestales en un vertisol en Nicaragua. Pp. 129132. In: Actas de los Simposios sobre Técnicas de producción de leña en fincas pequeñas y Recuperación de sitios degradados por medio de la silvicultura intensiva. Actas... Turrialba, CATIE, Costa Rica.

Drumond, M. A \& Oliveira, V. R. de. 1998. Introducción y selección de especies arboreas forrajeras en el área semiárida del Estado de Pernambuco, Brasil. In: Anais do I Congreso Latinoamericano IUFRO. El manejo sustentable de los recursos florestales: desafio del sigloAnais. Valdivia: IUFRO/CONAF. Tema 4. CD-Rom.

EMBRAPA. Centro de Pesquisa Agropecuária do trópico Semi-Árido, Petrolina,PE 1979. Relatório técnico anual do Centro de Pesquisa Agropecuária do Trópico SemiÁrido 1977-1978. Embrapa-DID, Brasília.

Goor, A. Y. \& Braney, C. W. 1976. Forestry planting in arid zones. 2th. ed. The Ronald Press Company, New York.

Guimarães Filho, C. \& Soares, J. G. G. 1988. Goat reproductive performance as affected by stocking rate on caatinga vegetation in the semiarid Northeast Brasil. Pp 351-357 In: Proceedings of International Research and Development Conference Arid Lands: Today and Tomorrow. Proceedings... Bouder: Westview Press, Tucson, Arizona.

Hughes, C. E.; Ochoa, O. \& Ponce, O. V. 1985. Especies nativas con potencial para la producción de leña en 
Centroamérica. Pp. 91-114. In: Actas de los Simposios sobre Técnicas de producción de leña en fincas pequeñas y Recuperación de sitios degradados por medio de la silvicultura intensiva. Actas... Turrialba, CATIE, Costa Rica.

Hughes, C. E. 1988. Recoleccion de semillas de Gliricidia sepium para ensayos internacionales de procedencia. Recursos Geneticos Forestales 16: 37-43

Lima, P. C. F. 1982. Comportamento de Leucaena leucocephala (Lam.) de Wit. comparado com Prosopis juliflora (SW.) DC. e Eucalyptus alba Reino ex Blume em Petrolina (PE), região semi-árida do Brasil. Dissertação de Mestrado. Universidade Federal do Paraná, Curitiba.

Lima, P. C. F. 1986. Usos múltiplos da leucena: produtividade no semi-árido brasileiro. Silvicultura 11(41): 55-57.

Lima, P. C. F.; Drumond, M. A.; Souza, S. M. de \& Lima, J. L. S. de. 1978. Inventário florestal da fazenda Canaã. In: Anais do III Congresso Florestal Brasileiro. Anais... Manaus-AM. Silvicultura 2(14): 398-399.

Martinez H. H. A. 1985. Producción de leña en la zona seca de Guatemala. Pp.77-89. In: Actas de los Simposios sobre Técnicas de producción de leña en fincas pequeñas y Recuperación de sitios degradados por medio de la silvicultura intensiva. Actas... Turrialba, CATIE, Costa Rica.
Picado V. W. 1985. Comportamiento de Gliricidia sepium (Jacq.) Steud en plantaciones por siembra directa en Guanacaste, Costa Rica. Pp 191-198. In: Actas de los Simposios sobre Técnicas de producción de leña en fincas pequeñas y Recuperación de sitios degradados por medio de la silvicultura intensiva. Actas... Turrialba, CATIE, Costa Rica.

Silva, H. D. da; Pires, I. E.; Ribaski, J.; Drumond, M. A.; Lima, P. C. F.; Souza, S. M. de \& Ferreira, C. A. 1980. Comportamento de essências florestais nas regiōes áridas e semiáridas do nordeste: resultados preliminares. EMBRAPA-DID, Brasília.

Stewart, J. L.; Dunsdon, A. J.; Hellin, J. J. \& Hughes, C. E. 1992. Wood biomas estimation of Central Americam dry zone species. Oxford Forestry Institute (Tropical Forestry Paper, 26), Oxford.

Tavares, S.; Paiva, F. A. F.; Tavares, E. J. \& Lima, J. L. S. de. 1969. Inventário florestal do Ceará - Estudo preliminar das matas remanescentes do município de Quixadá. Boletim de Recursos Naturais 7: 93-113.

Tavares, S.; Paiva, F. A. F.; Tavares, E. J. de; Carvalho, G. H. de \& Lima, J.L. S. de. 1970. Inventário florestal de Pernambuco - Estado preliminar das matas remanescentes dos municípios de Ouricuri, Bodocó, Santa Maria da Boa Vista e Petrolina. Boletim de Recursos Naturais 8: 149-193. 